\title{
Carnets
}

Revue électronique d'études françaises de l'APEF

Deuxième série - 9 | 2017

Reconnaissances et légitimité en français

\section{Légitimation du répertoire théâtral français sur la scène portugaise}

Ana Clara Santos

\section{(2) OpenEdition}

Journals

Édition électronique

URL : http://journals.openedition.org/carnets/2087

DOI : $10.4000 /$ carnets. 2087

ISSN : 1646-7698

\section{Éditeur}

APEF

\section{Référence électronique}

Ana Clara Santos, «Légitimation du répertoire théâtral français sur la scène portugaise », Carnets [En ligne], Deuxième série - 9 | 2017, mis en ligne le 31 janvier 2017, consulté le 02 mai 2019. URL : http:// journals.openedition.org/carnets/2087; DOI : 10.4000/carnets.2087

Ce document a été généré automatiquement le 2 mai 2019.

\section{(c) (i) (8)}

Carnets est mis à disposition selon les termes de la licence Creative Commons - Atribution - Pas d'utilisation commerciale 4.0 International. 


\title{
Légitimation du répertoire théâtral français sur la scène portugaise
}

\author{
Ana Clara Santos
}

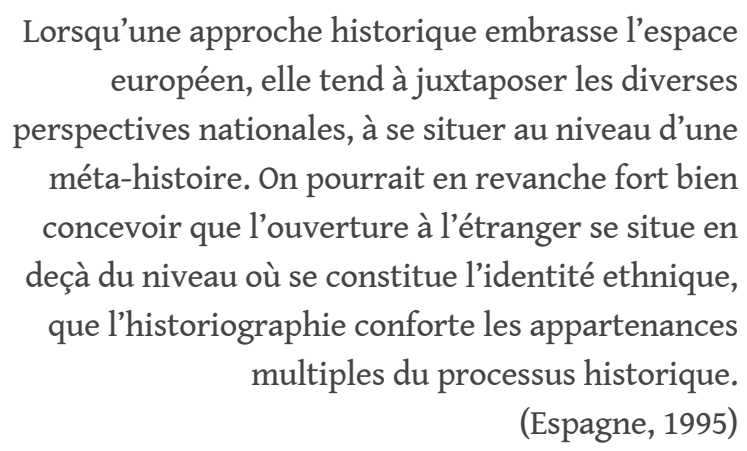

\section{Introduction}

1 Au cours de ces dernières décennies, les études comparées et les études culturelles ont développé un certain nombre de concepts au sein de l'approche des relations culturelles sur le plan international. Représentations, images, circulations, trajectoires, médiations, transferts et réseaux, entre autres, ont dicté une nouvelle orientation de la tradition de l'historiographie nationale des littératures et des cultures. L'étude de l'imagologie et des représentations de l'étranger ${ }^{1}$ promue par les comparatistes Daniel Henri Pageaux (1989, 2016) et Jean-Marc Moura $(1998,2016)$ obligent, d'une part, à déplacer le regard et à prendre en compte les fluctuations entre le champ de production (culture de départ) et le champ de réception (culture d'arrivée) et, de l'autre, à intégrer une autre figure dans le processus d'importation, celui du médiateur, véritable passeur entre cultures. Dans ce sens, aborder l'étude de l'altérité culturelle c'est avant tout intégrer les contextes de production de l'objet culturel et y déceler ce que Moura appelle «l'imaginaire social» (Moura, 1998).

2 Or les études menées dans le domaine du comparatisme des dernières années n'apportent pas une vision d'ensemble des relations profondes qui étayent les «effets de miroir» 
entre la France et les aires culturelles ibériques. Une approche de ces liens implique avant tout la construction d'une histoire européenne commune dans laquelle le rapprochement des histoires nationales serait complété par les effets, sur le plan international, des transferts culturels. Comme affirme Michel Espagne :

Le terme transfert culturel marque un souci de parler simultanément de plusieurs espaces nationaux, de leurs éléments communs, sans pour autant juxtaposer les considérations sur l'un et l'autre pour les confronter, les comparer ou simplement les cumuler. Il signale le désir de mettre en évidence des formes de métissage souvent négligées au profit de la recherche d'identités, d'une recherche qui vise naturellement à occulter ces métissages, même lorsque les identités en résultent (Espagne, 1999: 1).

De notre point de vue, si nous associons les enjeux de l'approche de la représentation de l'étranger et ceux de l'approche des transferts culturels et si nous arrivons à les croiser, au sein de l'étude de la circulation internationale, avec les mécanismes définis par Pierre Bourdieu² autour des opérations de "sélection» («qu'est-ce qu'on traduit? qu'est-ce qu'on publie? qui traduit? qui publie?»), de «marquage» («maison d'édition, collection, traducteur, préfacier ») et de «lecture » (Bourdieu, 1989 [2002]) nous aurons réussi à suivre les trajectoires des biens culturels, d'un espace culturel à un autre, et à leur assigner une signification autre conditionnée par le contexte historique et contemporain du champ d'accueil. Cela nous permet alors de parler de délocalisation, de réactualisation, d'assimilation ou de rejet car, comme explique Bourdieu, le sens et la fonction d'une œuvre étrangère sont déterminés au moins autant par le champ d'accueil que par le champ d'origine. Premièrement, parce que le sens et la fonction dans le champ originaire sont souvent complètement ignorés » (Bourdieu, 1989 [2002] : 4).

4 Il est donc important de s'attacher à la découverte de ces figures médiatiques entre les deux cultures, à ces passeurs de culture, et au rôle qu'ils jouent dans le processus de construction des identités nationales en matière culturelle au contact avec la différence et la nouveauté, c'est-à-dire, avec l'Autre.

\section{Structures et enjeux de légitimation}

5 La tradition philosophie et intellectuelle française déployée depuis la Révolution française, symbole de l'esprit des Lumières et de la révolution la plus universelle de la Modernité, est à l'origine de l'élan culturel suscité dans toute l'Europe par la capitale française à l'aube du Romantisme. Il n'en va pas autrement dans le domaine du théâtre. Bien au contraire. Les troupes françaises commencent à s'exporter à l'étranger et à se fixer dans d'autres capitales européennes. Il ne s'agit pas encore du phénomène lié aux tournées qui feront fureur dans la seconde moitié du siècle et qui rempliront les salles de spectacle un peu partout en Europe pour voir les acteurs et actrices françaises à la renommée internationale. Il s'agit d'un phénomène artistique encore plus important dans l'espace européen auquel la recherche n'a pas accordé jusqu'à maintenant l'attention méritée mais qui constitue, dans le cas portugais, le moteur essentiel de la rénovation de la dramaturgie nationale et des répertoires des salles de spectacle à Lisbonne.

Selon Michel Espagne, une capitale littéraire c'est une ville où « vivent de nombreux auteurs, où leurs livres sont produits, où leur consécration se décide, où sont engagées les traductions, où s'opère la diffusion et se pratique intensément la lecture critique " (Espagne, 2002: 323). L'image forgée de Paris depuis le siècle des Lumières correspond 
sans doute à cette acception : une capitale européenne, symbole de diffusion des idées et des modèles littéraires et culturels. Dans ces circonstances, il était tout à fait attendu que les artistes portugais ouvrent massivement les portes à cette importation, d'autant plus que celle-ci était facilitée par les conditions d'accessibilité linguistique, le français étant la première langue étrangère dans le pays. S'il est vrai que la langue a constitué un vecteur du passage transnational en matière littéraire, elle représentait sur la scène lisboète une des principales prémisses pour la permanence aussi longue d'une troupe étrangère sur le sol national portugais (1834-1837).

7 A l'image de ce qui arriva dans d'autres pays européens, la culture française a occupé une place prépondérante dans la formation intellectuelle et culturelle au Portugal depuis le $\mathrm{XVIII}^{\mathrm{e}}$ siècle. La répression instaurée par le marquis de Pombal, ministre du roi D. José I (1750-1777) et l'action menée par la Real Mesa Censória, qu'il créa en 1768, ne furent pas suffisantes pour contrecarrer l'influence, sur le plan idéologique, liée à la circulation des biens culturels venus de Paris. En effet, malgré la censure, les idées de la Révolution française et celle des grands philosophes des Lumières, ainsi que certains modèles littéraires et dramatiques du XVII ${ }^{e}$ et du XVIII ${ }^{e}$ siècles ont intégré les débats intellectuels et les circuits de production artistique. Même si cet enthousiasme a été refroidi quelque peu, à l'aube du XIX ${ }^{e}$ siècle, à cause des invasions napoléoniennes et des troubles politiques nationaux, ce qui est vrai c'est que l'engouement pour tout ce qui était français n'arrêta pas de croître au cours du siècle. Le pays, ayant recouvert une plus grande stabilité culturelle et sociale après les invasions françaises $(1807-1810)^{3}$ et, surtout, après la fin de la guerre civile qui opposa libéraux constitutionnels et absolutistes (1820-1834), connait, à partir de l'année 1834, année de l'arrivée de la troupe française d'Emile Doux qui se fixe pendant deux ans dans le petit théâtre Rua dos Condes, une période de reconstruction à tous les niveaux, y compris au niveau culturel et artistique.

8 Du point de vue littéraire et théâtral, les premières décennies du XIX siècle coïncident au Portugal, comme dans d'autres pays européens, avec une période avec laquelle prime la prise de conscience de l'urgence de l'édification d'une culture nationale. Grâce à la révolution romantique entamée par les écrivains Almeida Garrett, Alexandre Herculano et bien d'autres, la vie artistique s'intensifie autour de nouveaux enjeux et nouvelles pratiques sociales et culturelles. À l'issue des luttes libérales et nationalistes, l'un des enjeux majeurs est sans doute celui d'un nouveau concept de nation, fortement ancré sur l'idée d'un patrimoine national, légitimé par un passé commun. Nous assistons alors à des efforts consubstantiels en vue de la mise en place d'un système d'instruction publique élargie et des structures d'accessibilité à la culture plus efficaces.

9 Au niveau des infrastructures et de la dynamisation de la représentation théâtrale, la construction de nouveaux théâtres ${ }^{4}$ est la preuve majeure d'une réforme profonde voulue par la reine D. Maria II et le roi D. Fernando. Les monarques ont vite compris l'urgence pour le royaume de s'assimiler aux autres cours européennes du point de vue de la vie artistique préconisée dans la capitale. Restait alors le problème de l'efficacité au niveau de la performance. Une fois les édifices architecturaux bâtis aux conditions de représentation excellentes et jamais vues dans la capitale - si on fait exception, bien sûr, du fameux théâtre lyrique S. Carlos - il fallait tout faire pour le rendre digne d'un théâtre à la mode, digne des autres capitales européennes : former les acteurs afin de constituer une troupe nationale et un nouveau répertoire. Là-dessus, comme nous l'avons déjà démontré à plusieurs reprises, le contact avec l'étranger et le rôle joué par les artistes français à Lisbonne, notamment par Emile Doux et l'acteur Paul au Conservatoire et à 
l'école dramatique ainsi que dans quelques troupes d'acteurs, contribua à la mise en place des structures de formation artistique et à sa légitimité dans le champ culturel portugais. Les nouvelles structures créées (le Conservatoire général de l'Art dramatique, divisé en une École dramatique ou de déclamation, une École de musique et une École de danse, mimique et gymnastique spéciale ; le Théâtre national D. Maria II, le Théâtre D. Fernando) produisent un discours de légitimation de certaines pratiques culturelles liées au culte du patrimoine culturel national. La culture légitime -- celle qui est promue par l'Etat, ses structures et agents influents ${ }^{5}$-- instaure, sous l'impulsion étrangère, le culte du drame historique. Cette «culture légitime dominante " (Lahire, 2004) sur le plan de la création littéraire a toujours été remise en cause sur scène par les faiseurs de théâtre à Lisbonne. Les praticiens, metteurs en scène et traducteurs à la tête, se dressent contre cette culture littéraire en la remplaçant par un autre modèle qui, à leurs yeux, constitue une autre forme de consécration avant-gardiste sur la scène : le vaudeville.

La dilution de la légitimité du drame historique romantique sur la scène portugaise est due à la concurrence accrue des répertoires des dits théâtres de second ordre, celui du théâtre Rua dos Condes et celui du Salitre, fortement ancrés, dans leurs pratiques, sur les répertoires importés, imités et adaptés des théâtres de Boulevard parisiens. Comment interpréter ces données devenues publiques au début du $\mathrm{XxI}^{\mathrm{e}}$ siècle depuis que l'on a publié les premiers répertoires de théâtre au $\mathrm{XIX}^{\mathrm{e}}$ siècle au Portugal (Santos, Vasconcelos, 2007, 2011)?

Grâce aux approches sociologiques, on sait que l'affrontement symbolique participe à la légitimité. L'analyse des fluctuations du champ symbolique fait apparaitre des structures de classement par lesquelles les agents saisissent le monde socioculturel. $\mathrm{Si}$ «ces classements sont des enjeux essentiels de la lutte pour la légitimation de la domination [et] enferment l'affrontement symbolique dans les limites qui définissent le domaine des représentations possibles ", ils constituent essentiellement un instrument de mesure de la « discontinuité » de positions et de stratégies dans le champ. Légitimer une domination suppose des dominés et implique, d'emblée, que « le champ soit accepté comme champ de lutte concurrentielle par les dominés; ensuite, que l'enjeu de cette lutte soit l'appropriation différentielle de ce que le champ produit comme capital symbolique; troisièmement, que les dominés partent avec un handicap insurmontable; enfin, que le handicap soit masqué » (Haro, 1980 : 1183).

Deux réseaux culturels différents sont à la base de ce processus de légitimation de la matière théâtrale importée des théâtres de Boulevard parisiens. D'un côté, se trouve le réseau de l'appareil de l'Etat (théâtre national, Conservatoire...) et les écrivains romantiques (Almeida Garrett, Alexandre Herculano, entre autres) qui légitiment, par leurs décisions ${ }^{6}$, le modèle du drame romantique en vue de la rénovation de la littérature nationale. De l'autre côté se situe le réseau constitué par les troupes, les imprésarios et les metteurs en scène, véritables décideurs des nouvelles tendances de la scène portugaise. Á une dramaturgie à vocation littéraire s'oppose une dramaturgie à vocation théâtrale et scénique susceptible de répondre aux besoins d'une classe sociale croissante, forte consommatrice de biens culturels, la classe bourgeoise. Déceler l'affrontement symbolique en vue de la légitimité culturelle c'est appréhender le fonctionnement du champ symbolique afin de rendre visibles les différentes positions et stratégies mises en place.

13 Dans cette perspective, les défis pour l'historien du théâtre sont énormes et cela explique peut-être en partie que l'histoire du théâtre national n'ait pas intégré, jusqu'à 
aujourd'hui, cette double dimension du regard porté sur ce qui est écrit pour le théâtre et ce qui est joué dans les théâtres.

\section{Effets de médiation et opérations de légitimation}

14 Il est bien évident que les biens culturels transposés, en l'occurrence le répertoire théâtral parisien, n'occupe plus la même place dans l'autre contexte national (portugais) et qu'il bénéficie très souvent d'une autonomisation qui lui advient du processus d'adaptation pour répondre à un « imaginaire social » puisque « la référence étrangère est intégrée à un débat propre au contexte d'accueil (...) et n'est plus déterminée que par les positions des protagonistes du débat en cours » (Espagne, 1999 : 23).

C'est dans cette perspective que nous situons l'étude que nous menons, depuis quelques années, sur les relations luso-françaises en matière théâtrale. Si nous avons justement commencé ces recherches par l'étude de la fortune des dramaturges classiques français au Portugal avec le souci de déterminer la distance ou la fidélité par rapport à l'original au sein du processus d'importation culturelle, une autre approche du contact avec l'étranger nous semblait prioritaire pour accéder à une connaissance plus effective de ses effets sur la culture nationale. Il est urgent de déterminer par quels moyens les contacts avec la France littéraire et théâtrale permettent de constituer non seulement une identité nationale mais aussi l'édification une réforme du théâtre national. Il ne s'agit pas, dans cette approche, de se limiter à des prélèvements de données des pièces et auteurs importés, mais de tracer une esquisse des circuits de circulation et d'appropriation qui légitiment l'instauration de certains modèles étrangers. Il s'agit donc d'opérer un déplacement du regard et de voir comment la culture nationale joue le jeu du rapprochement ou de l'écartement de l'Autre.

Du processus d'importation, défini par José Lambert (1986) et Pascale Casanova (1999) au service la rénovation des littératures et des cultures, découle l'analyse des différentes attitudes du champ littéraire et culturel face à l'Étranger, ainsi que l'évolution de la pratique de la traduction, sortie de sa sphère dite "marginale ", pour mieux se situer dans le débat de la construction de l'identité nationale et dans le processus de reconnaissance et de légitimité de l'Autre :

[La traduction] est une forme de reconnaissance littéraire et non un simple changement de langue, pur échange horizontal qu'on pourrait (devrait) quantifier pour connaître le volume des transactions éditoriales dans le monde. La traduction est au contraire l'enjeu et l'arme majeure de la rivalité universelle entre les joueurs, une des formes spécifiques de lutte dans l'espace littéraire international, instrument à géométrie variable (Casanova, 1999 : 188).

Or, si la traduction est la «grande instance de consécration spécifique de l'univers littéraire » (Casanova, 1999 : 188), il importe d'analyser, en matière théâtrale, par quelles instances l'importation de la dramaturgie française se retrouve légitimée sur la scène portugaise. $\mathrm{Au}$ XIX ${ }^{e}$ siècle, ce processus se retrouve condensé surtout par la presse et des rituels de consécration, concentrés autour des tournées théâtrales, des collaborations artistiques bilatérales ainsi que la circulation de certaines collections éditoriales. L'expérience du contact avec la France assurera, d'un côté, le rayonnement et la reconnaissance d'une certaine culture dramatique française au Portugal et permettra, de l'autre, de mesurer l'effet des activités de transfert culturel sur l'évolution des relations interculturelles luso-françaises afin de resituer la dimension de la médiation culturelle de 
certains agents portugais. Que signifie alors traduire une pièce au théâtre? S'agit-il uniquement de transfert culturel et de diffusion d'un répertoire ou s'agit-il, au contraire, de légitimer un certain répertoire sur le panorama culturel national ?

Les conditions socio-économiques, politiques et culturelles du début du siècle favorisent le surgissement d'une élite intellectuelle qui prendra en main le délinéament de la pratique et la gestion culturelles. L'implémentation de certains circuits, réseaux et espaces de représentation théâtrale aboutit, comme nous venons de voir, à la constitution de lieux et de formes de fixation d'une mémoire culturelle française dans la capitale portugaise. Nous revenons ici sur le concept de «champ littéraire » de Pierre Bourdieu afin de mieux cerner «le rapport de force entre les agents ou les institutions engagés dans la lutte ou, si l'on préfère, de la distribution du capital spécifique qui, accumulé au cours des luttes antérieures, oriente les stratégies ultérieures»(Bourdieu, 1992: 144). Dans ce sens, la traduction théâtrale est mise au service de la représentation scénique car elle sert, en premier chef, la création d'un spectacle autre, c'est-à-dire adapté au public de la culture d'arrivée. Par le bais du processus de transmutation, elle se veut une nouveauté par rapport aux tendances de la scène nationale.

le sait, l'importation d'œuvres étrangères traduites a presque toujours causé des conflits et des tensions dans le champ littéraire et culturel d'accueil. Quel statut est-il perpétué entre l'œuvre traduite et son original ?

Les moyens par lesquels les traducteurs au service des troupes portugaises sur la scène lisboète adaptent une grande partie du répertoire joué par la troupe française entre 1835 et 1838 est révélateur du rôle assigné à la traduction considérée alors comme vecteur des valeurs de la culture théatrale française et de revendication de modèles innovants, inexistants auparavant dans la culture portugaise. C'est donc par le caractère innovant que la traduction sert l'objectif de la rénovation des répertoires des troupes portugaises. Or si l'innovation est mise en avant, la provenance étrangère de cette innovation est effacée et l'Autre, c'est-à-dire le créateur français, est rarement nommé dans les annonces des spectacles et les éditions des artistes portugais du XIX ${ }^{e}$ siècle. Les processus d'« accommodation » (Moura, 1998: 51) et d'adaptation par lesquels se trouve engagée la circulation des pièces françaises sur la scène lisboète, travesties par le changement des titres, des noms des personnages et des lieux de l'intrigue, sont notoires des valeurs qu'on leur investi dans le processus de légitimation. Dans un champ culturel où la production d'œuvres originales était la voie la plus reconnue vers la légitimation de la production théâtrale et de la constitution des répertoires nationaux, il est compréhensible qu'on essaie d'escamoter la production véhiculée par la traduction, surtout à un moment où les troupes théâtrales entrent en concurrence et veulent légitimer leur position. Bien que cette concurrence soit une constante au $\mathrm{XIX}^{\mathrm{e}}$ siècle et qu'elle reste vivante au cours de la deuxième moitié du siècle avec la prolifération des salles de spectacle à Lisbonne, elle a connu ses débuts après le départ de la troupe française d'Emile Doux en 1837 et a été fixée par sa diffusion dans la presse de l'époque. En effet, les revues $O$ Desenjoativo et Atalaia dos Theatros font écho, presque au jour le jour, des tensions entre la troupe du théâtre Rua dos Condes et celle du théâtre du Salitre. L'enjeu lié à ces tensions traverse le siècle entier et met en opposition un répertoire national, qui se veut original, libéré du joug étranger, avec un répertoire traduit du répertoire parisien qui gagne, de plus en plus, la faveur du public. L'enjeu était de taille. Il a permis, très tôt, la création d'un véritable laboratoire d'expérimentations des nouvelles pratiques théâtrales marquées surtout par l'innovation du drame et du vaudeville français. La multiplication des salles de spectacles, la 
constitution des répertoires qui accompagnaient surtout les tendances des théâtres de Boulevard parisiens, les revues spécialisées en dramaturgie et les collections éditoriales nourries par la traduction du répertoire parisien assurent la naissance d'un marché du théâtre en pleine croissance au cours du siècle. Or dans ce nouveau marché, tout comme dans n'importe quel champ symbolique, s'instaure un rapport de forces entre la culture dominante et la culture importée. La submersion de la scène par la présence étrangère alliée à l'excès du nombre de traductions mises en scène sur les théâtres lisboètes est à l'origine d'une forte contestation, du début jusqu'à la fin du siècle, au nom du théâtre national :

Il y a longtemps que le théâtre portugais vit de traductions de pièces françaises plus ou moins détestables, généralement mal interprétées et presque toujours inadaptées à notre scène parce qu'elles n'ont aucun rapport avec les traditions et les mœurs de la société portugaise. On se limite à attirer le public par la complexité de l'action, par les dénouements extraordinaires, par la beauté du scénario ou par les costumes voyants et extravagants (Bastos, $1884: 20$ ). ${ }^{\text {? }}$

21 À titre d'illustration, prenons le cas de deux dramaturges les plus joués sur la scène portugaise de l'époque. Comme un peu partout en Europe, Eugène Scribe fut le dramaturge le plus joué et le plus adapté aux répertoires des salles de spectacle portugais. Son nom bénéficiait d'une telle renommée à Lisbonne que la critique portugaise arrive même à présenter certains dramaturges au cours de la deuxième moitié du siècle en liaison avec son système dramatique. Ce fut le cas de Victorien Sardou qui bénéficia, lui aussi, d'une consécration et d'une popularité sur la scène portugaise, à partir des années 60, qui le met au même rang d'Émile Augier ou d'Alexandre Dumas fils. Là encore, tout comme dans le cas de Scribe, le processus de légitimation est similaire et prend assise sur le même type de médiateurs : les troupes étrangères et nationales. Les premières, venues de la France ou de l'Italie, passent par Lisbonne en tournée -- la plupart du temps en chemin vers l'Amérique du sud - et permettent au public portugais, avide de nouveautés théâtrales, de découvrir avec quelques semaines d'intervalle, voire quelques mois, les grands succès de la scène parisienne. Parmi ces troupes nous comptons des artistes qui jouissaient d'une forte renommée en Europe comme, par exemple, la Marini, la Duse, Emmanuel, Novelli. Zampieri, Céline Chaumont, Réjane et, bien sûr, Sarah Bernhardt. Parmi les secondes, deux troupes ont vite intégré les pièces du dramaturge français dans leur répertoire : la troupe Rosas \& Brasão, la troupe du théâtre national D. Maria II, et la troupe de Lucinda Simões. Cette dernière jouera encore Fédora à Lisbonne en 1884, La Tosca en 1893, Madame Sans-Gêne en 1895 et Georgette en 1896. L'un des plus grands succès sur la scène portugaise est sans doute celui de Madame Sans-Gêne lors de la réouverture du théâtre Rua dos Condes le 9 novembre 1895. Après la représentation théâtrale, une des revues spécialisées dans le domaine, la Revista Theatral, consacre un numéro extraordinaire au dramaturge français et à sa dramaturgie. Publié dans la rubrique des "Célébrités étrangères », un dossier important qui réunit plus d'une quinzaine de pages lui est dédié avec, entre autres, la liste de la distribution de Madame Sans-Gêne au théâtre du Vaudeville à Paris accompagnée des caricatures de Lérand, Grisez et Dorval dans les rôles de Fouché, Vinaigre et Roustan ; un portrait du dramaturge et une photo des essais de la pièce qui renforce le rôle du dramaturge en tant que metteur en scène; la traduction en portugais des extraits de critiques portées sur la pièce comme celles de Francisque Sarcey, de Jules Lemaître, de Camille Bellargue et d'Amedée Mérandat. Aux yeux de l'un des directeurs de la revue, Collares Pereira, cette première représentation portugaise de Madame Sans-Gêne à partir de la traduction de Moura Cabral, reste sans 
doute l'un des grands succès du nouveau théâtre Rua dos Condes grâce à la force dramatique de la pièce, la richesse de la mise en scène et le jeu des acteurs. Selon le critique portugais, si ce succès se complait dans les différentes facettes de sa réception auprès de quelques salles de spectacle européennes, il se doit surtout, sur le théâtre portugais, au rôle joué par la troupe de Lucinda Simões ainsi que le savoir-faire d'une actrice qui avait côtoyé de près avec les grands modèles parisiens :

(...) L'intérêt qu'elle éveille n'est pas le même auprès de chaque auditoire: en France on a apprécié surtout dans Madame Sans-Gêne la partie anecdotique de la vie de Napoléon, l'exactitude du milieu, la fidélité de la copie du cabinet impérial, l'authenticité des objets qui le décoraient, l'évocation de cette époque glorieuse ; à Londres, au Garrick, dans sa tournée au cours de cette saison, Réjane a cueilli de forts applaudissements, mais le succès de la pièce était principalement dû à l'intrigue ; ici, il m'a semblé que c'est la partie dramatique de la pièce qui a attiré l'attention. C'est Lucinda Simões qui, de plein droit, a joué le rôle principal, celui de la blanchisseuse transformée grâce aux hasards de la guerre et de la fortune en duchesse et maréchale de la France. Ayant vu à Paris la créatrice du personnage, l'actrice portugaise ne s'est pas éloignée de son modèle, et par conséquent, en ce qui concerne le dessin du type, la critique n'aura pas à faire des remarques car, bon ou mauvais, exact ou inexact, il est tel que l'auteur l'a dessiné (Pereira, 1895).

Du côté de l'activité de la troupe Rosas \& Brasão, fixée au théâtre national D. Maria II, l'année 1882 coïncide avec un intérêt plus accentué envers la dramaturgie de Sardou. Quelques mois seulement séparent les représentations d'Odette (1881) et de Fédora (1882) au théâtre du Vaudeville à Paris et la représentation des deux pièces à Lisbonne. Augusto Rosa qui connait bien le théâtre de Sardou raconte, à ce propos, avec quelle émotion il assiste à la reprise de la Tosca au théâtre de la Porte Saint-Martin au cours du mois de septembre 1889 et quel plaisir inouï il a non seulement de rencontrer Sarah Bernhardt mais surtout de faire la connaissance de Victorien Sardou au moment où celui-ci rend visite à l'actrice dans sa loge :

C'était le mois de janvier de cette année-là [1889] et la grande et irremplaçable star [Sarah Bernhardt] allait faire sa réapparition au théatre de la Porte Saint-Martin dans Tosca, un des plus grands triomphes, qu'elle avait représenté l'année précédente à Lisbonne lorsqu'elle alla en tournée au Portugal (...) Sarah, cette nuitlà, était admirable; le repos qu'elle avait pris pendant quelque temps lui avait donné une énergie extraordinaire. Toute la grande scène de torture au $3^{e}$ acte était faite avec brio, une puissance et une richesse de tons inégalables. A la fin de cet acte on alla dans sa loge ; avec beaucoup de difficultés, mais on a fini par y arriver (...) lorsqu'on allait entamer la conversation, apparait à la porte un petit homme mince, au visage rasé, aux cheveux longs et au regard espiègle. C'était Sardou, le grand Sardou, qui allait visiter l'interprète géniale de ses dernières pièces. Sarah Bernhardt se leva avec le plus grand respect et, d'un joli mouvement gracieux, s'inclina devant Sardou lui disant: -- Cher maitre, vous m'honorez beaucoup, venant me dire bonsoir. Le petit grand homme de théâtre, s'inclinant à son tour, lui baisa la main et entama la conversation. Quelques moments après, Sarah nous présenta, au milieu de galantes éloges, à Sardou en tant que ses collègues portugais et lui, nous serrant la main comme si on était de vieux amis, de faire à notre pays les plus nobles démonstrations. Cette visite à la loge de Sarah et cette présentation sont restées gravées dans notre mémoire dont elles ne s'éteindront jamais (Rosa, $1917:$ 78-80).

Le côtoiement des artistes portugais avec les artistes français à Paris était devenu une constante au cours de la deuxième moitié du siècle. À l'importation des modèles traduits pratiquée au début du siècle s'ajoute l'importation d'un savoir-faire au niveau de la mise en scène. 

provoqué, à la suite des événements parisiens, un tumulte réactionnaire contre Victorien Sardou, mais que certains critiques, comme Caetano Pinto, s'empressent de défendre :

Sur Odette il y a eu une lutte héroïque d'adjectifs, un tournoi violent de phrases, qui a fait sortir dans l'arène les combattants les plus hardis de la critique dramatique (...) S'il y a quelque chose digne de sensation dans le jugement d'une pièce c'est le nom de l'auteur. Sardou a droit incontestablement à la vénération de tout le monde, de tous ceux qui comprennent quelque chose au théâtre. Il est un réactionnaire. Sa galerie de personnages est une vitrine de colporteur, mais les thèses de ses pièces sont un cautère des vices sociaux : La beauté du dialogue et la verve y répandue, surélèvent, peut-être, les plus grands défauts de sa vie dramatique. Mais Sardou est un plagiaire -- nous a répondu, il y a quelques mois, Paris dans ses chroniques du théâtre français -- est un bi-plagiaire de Maria Uchard et de Giacommetti. Et la chronique à Lisbonne qui veut égaler la chronique française en matière de scandale, vociféra contre Sardou toute l'insolente adjectivation arrivant même à le comparer à un copiste de théâtre (Pinto, 1882).

Cet exemple est révélateur d'une pratique culturelle de l'époque et qui prétend légitimer l'importation du modèle théâtral et dramatique français. La presse de l'époque constitue effectivement un vecteur de légitimation grâce à la multiplication d'extraits des pièces des plus grands dramaturges français devenus célèbres sur la scène européenne. A cela s'ajoute la diffusion de l'annonce des dernières représentations sur les théâtres parisiens, quelques critiques dramatiques françaises que l'on traduit en portugais et l'introduction de documents iconographiques illustratifs des auteurs et des scènes dramatiques les plus célèbres à Paris. Citons, à titre d'exemple, la revue portugaise Revista Theatral et la revue franco-lusophone A Illustração, dirigée à Paris par Mariano Pina et diffusée au Portugal et au Brésil. Au cours des deux décennies du XIX ${ }^{\mathrm{e}}$ siècle alors que le théâtre de Sardou connait un indice de popularité croissante, on multiplie les allusions à son système dramatique et les photos qui consacrent une pratique littéraire et théâtrale. Le goût prononcé envers le répertoire de son théâtre créé sous la Troisième République, consacre sa carrière sur la scène péninsulaire et le met au goût du jour d'un public bourgeois ascendant, coïncidant, du moins dans les lettres portugaises, avec un renouveau du théâtre historique ou plus précisément avec ce qu'on appelait à l'époque le «drame d'actualité ». Véritable héritier de Scribe toujours à l'écoute des attentes du public il a su conquérir bien au-delà de l'Hexagone un public avide de fresques historiques et, en même temps, au goût prononcé envers un théâtre adapté à l'actualité et aux mœurs de l'époque.

\section{En guise de conclusion}

Dans le domaine de l'historiographie théâtrale au Portugal, les historiens de théâtre ont surtout privilégié une étude de l'histoire du texte dramatique produit par les auteurs nationaux et ont complètement condamné à l'oubli les répertoires des salles des spectacles et la vie artistique liée à la représentation théâtrale. Or, il nous semble de plus en plus légitime et urgent d'étudier les opérations d'importation, de transmutations et d'adaptation effectuées par les agents culturels portugais sur les importations étrangères et, plus particulièrement, sur celles venues de Paris. Cette nouvelle donne permettra de remplacer le culte des grands auteurs cher aux études littéraires $\mathrm{du} \mathrm{xx}^{\mathrm{e}}$ siècle par la mise en perspective de circuits et réseaux culturels susceptibles de redimensionner le questionnement et la perception d'une histoire interculturelle. On l'a vu, La circulation des biens culturels entre les deux pays s'effectuent par l'intermédiaire de médiateurs 
individuels et collectifs (troupes, impresarios, traducteurs, metteurs en scène, maisons d'édition) et de stratégies de d'appropriation et d'autonomisation qui s'effectuent en continu par différentes instances de légitimation. Les nombreux artistes français qui s'installent dans les salles de spectacle à Lisbonne au du XIX ${ }^{e}$ siècle ont certainement joué un rôle crucial sur la pratique théâtrale nationale au Portugal. Leur médiation culturelle doit donc être prise au sérieux et valorisée au sein d'un système de légitimation et de reconnaissance littéraire et théâtrale qui privilégie les échanges culturels avec la France. Car, nous l'avons vu, cette médiation sert à renforcer la place centrale occupée par l'Autre, soit par un processus d'assimilation ou, au contraire, de rejet, dans la constitution de la conscience nationale. De plus, l'effet de miroir qu'elle instaure entre la scène parisienne et la scène portugaise n'est pas un simple effet réflecteur qui dure le temps de la tournée à Lisbonne mais instaure, au contraire, un effet durable sur la transmutation des répertoires de certaines troupes portugaises et les tendances du théâtre national.

\section{BIBLIOGRAPHIE}

BASTOS, Teixeira (1884). «O teatro moderno em Portugal », Revista de Estudos Livres.

BOURDIEU, Pierre (1992) [1980]. Questions de sociologie. Paris : Editions Minuit.

BOURDIEU, Pierre (2002) [1989]. « Les conditions sociales de la circulation internationale des idées ", Actes de la recherche en sciences sociales, vol. 145, décembre. La circulation internationale des idées. pp. 3-8

<http://www.persee.fr/doc/arss_0335-5322_2002_num_145_1_2793>

CARO, Jean-Yves (1980). « La sociologie de Pierre Bourdieu. Éléments pour une théorie du champ politique », Revue françaises de science politique, vol. 30, nº 6, pp. 1171-1197.

CASANOva, Pascale (1999). La République mondiale des lettres. Paris : Le Seuil.

ESPAGNE, Michel (1991). Les transferts franco-allemands. Paris : PUF.

ESPAGNE, Michel (1995). « Problèmes d'histoire interculturelle », Revue germanique internationale, nº 4 , pp. 5-24.

ESPAGNE, Michel (2002). « Les capitales littéraires allemandes », in Christophe Charles, Daniel Roche (dir.). Capitales culturelles, capitales symboliques. Paris et les expériences européennes. Paris : Publications de la Sorbonne, pp. 323-334.

ESPAGNE, Michel, (2005). « Les transferts culturels ». H-Soz-u-Kult, <http://

hsozkult.geschichte.hu-berlin.de/forum/2005-01-002>.

HABCHI, Sobhi (2007). Littératures et cultures en dialogue. Mélanges offerts à D- H. Pageaux. Paris :

L'Harmattan.

LAHIRE, Bernard (2004). La culture des individus. Dissonances culturelles et distinction de soi. Paris : La Découverte. 
LAMBERT, José (1986). « Les relations littéraires internationales comme problème de réception », in P. Borner, J. Riesz, B. Scholz (éd.). Sensus Communis. Contemporary Trends Comparative Literature. Tübingen : G. Narr.

LE HIR, Marie-Pierre (1992). Le romantisme aux enchères : Ducange, Pixérécourt, Hugo. Amsterdam/ Philadelphia : John Benjamins.

MOURA, Jean-Marc (2016). « De quelques usages littéraires des « étranges étrangers ", in Ana Clara Santos, Maria de Jesus Cabral. 2016. L'Etranger. Paris : Le Manuscrit, pp. 43-73.

MOURA, Jean-Marc (1998). L'Europe littéraire et l'ailleurs. Paris : PUF.

PAGEAUX, Daniel-Henri (2016). « La « dimension étrangère » comme champ de recherche en littérature générale et comparée » in Ana Clara Santos, Maria de Jesus Cabral. 2016. L'Etranger. Paris : Le Manuscrit, pp. 17-42.

PAGEAUX, Daniel-Henri (1989), «De l'imagerie culturelle à l'imaginaire », in Brunel \& Chevrel

(dir.). Précis de Littérature comparée. Paris : PUF, pp. 133-161.

PEREIRA, Collares (1895). « Madame Sans-Gêne », Revista Theatral, 2e série, année I, vol. 1, 끄 22, 15 novembre.

PINTO, Caetano (1882). « Sardou », Perfis artísticos, ํำ 10.

ROSA, Augusto (1917). Memórias e estudos. Lisboa : Livraria Ferreira.

SANTOS, Ana Clara, CABRAL, Maria de Jesus (2016). L'Etranger. Paris : Le Manuscrit.

SANTOS, Ana Clara, VASCONCELOS, Ana Isabel (2011). Repertório teatral na Lisboa oitocentista (1846-1852)

. Lisboa : Imprensa Nacional- Casa da Moeda.

SANTOS, Ana Clara, VASCONCELos, Ana Isabel (2007). Repertório teatral na Lisboa oitocentista (1835-1846)

. Lisboa : Imprensa Nacional- Casa da Moeda.

\section{NOTES}

1. Voir, à ce propos, le volume publié cette année sur l'Etranger dans la collection «Exotopies » chez Le Manuscrit (Santos, Cabral, 2016) auquel les deux spécialistes ont collaboré.

2. «Le transfert d'un champ national à un autre se fait à travers une série d'opérations sociales : une opération de sélection (qu'est-ce qu'on traduit? qu'est-ce qu'on publie ? qui traduit? qui publie ?) ; une opération de marquage (d'un produit préalablement "dégriffé ») à travers la maison d'édition, la collection, le traducteur et le préfacier (qui présente l'œuvre en se l'appropriant et en l'annexant à sa propre vision et, en tout cas, à une problématique inscrite dans le champ d'accueil et qui ne fait que très rarement le travail de reconstruction du champ d'origine, d'abord parce que c'est beaucoup trop difficile); une opération de lecture enfin, les lecteurs appliquant à l'œuvre des catégories de perception et des problématiques qui sont le produit d'un champ de production différent » (Bourdieu, 1989 [2002] : 4).

3. Au début du XIX ${ }^{\mathrm{e}}$ siècle, le Portugal, par les liens politiques et commerciaux qu'il maintenait avec l'Angleterre, représentait aux yeux de Napoléon Bonaparte un obstacle à la domination française absolue sur cette partie du territoire européen. Avant même la première invasion française (1807), le roi Jean VI transfère le siège du royaume portugais à Rio de Janeiro où la couronne et une grande partie de la cour seraient totalement en sécurité. Les invasions françaises (1807-1810) et cette stratégie politique et militaire portugaise marquent le début de la 
crise du secteur culturel et artistique national qui se prolonge encore pendant plus de deux décennies.

4. Nous rappelons, au passage, que le théâtre national D. Maria II et le théâtre D. Fernando, inaugurés, en 1846 et en 1849, respectivement, constituent, du point de vue de l'innovation architecturale et théâtrale, les deux pôles d'attraction théâtrale majeure dans la capitale portugaise au cours de la première moitié du XIX ${ }^{\mathrm{e}}$ siècle.

5. La critique portugaise a depuis longtemps reconnu le rôle majeur joué par Almeida Garrett, poète, romancier et dramaturge romantique qui, rentré de l'exil en Angleterre et en France, mena de plein pied la réforme du théâtre national autour de la création du drame romantique et de la formation d'une nouvelle génération d'auteurs.

6. Nous pensons ici surtout aux concours et aux prix attribués aux drames historiques par le Conservatoire royal.

7. Teixeira Bastos, «O teatro moderno em Portugal » in Revista de Estudos Livres, 1884, p. 20.

\section{RÉSUMÉS}

Mettre en avant la circulation des biens culturels (en l'occurrence, le répertoire théatral) et leur appropriation à travers les médiations individuelles et collectives entre la France et le Portugal au XIX ${ }^{\mathrm{e}}$ siècle c'est soulever la question de la légitimité au sein des transferts culturels. La traduction théâtrale tend, à cette époque, à sortir de sa sphère dite "marginale " afin de matérialiser le point de contact entre les deux cultures et dessiner les trajectoires de la création artistique nationale pour concrétiser la réforme des pratiques théâtrales et leur rénovation. Du processus d'importation découle l'analyse des différentes attitudes du système littéraire et culturel face à l'Étranger, ainsi que l'évolution de la pratique de la traduction au sein de la construction de l'identité nationale et de l'idée même de reconnaissance et de légitimité de l'Autre.

To bring the circulation of cultural assets (namely, the theatre repertoire) to the front as well as its appropriation through individual and collective mediations between France and Portugal in the nineteenth century implies bringing up the topic of legitimacy within cultural transfers. In those days the translation of theatre tends to escape the so called "marginal" sphere in order to materialize the contact point between the two cultures and to define the trajectories of the national artistic creation - so as to put the reform of theatre practices and their renewal into practice. From the process of importation stem the analysis of different attitudes of the literary and cultural system also confronting the foreigner, as well as the evolution of the practice of translation within the construction of a national identity and of the very idea of recognition and legitimacy of the Other.

INDEX

Mots-clés : légitimation, théâtre portugais, transfert culturel, étranger

Keywords : legitimation, Portuguese theatre, cultural transfer, foreigner 


\section{AUTEUR}

\section{ANA CLARA SANTOS}

Un. do Algarve/CET- Un. de Lisboa

avsantos[at]ualg.pt 